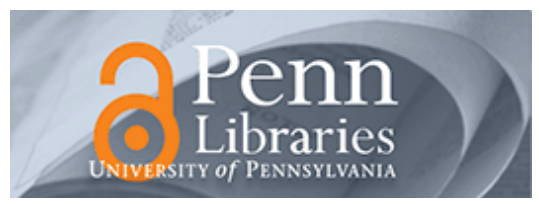

University of Pennsylvania ScholarlyCommons

3-16-2012

\title{
Avoiding Metallic Walls: Use of Modal Superposition in Plasmonic Waveguides to Reduce Propagation Loss
}

Francisco J. Rodríguez-Fortuño

University of Pennsylvania, Universidad Politecnica de Valencia

Nader Engheta

University of Pennsylvania, engheta@ee.upenn.edu

Follow this and additional works at: https://repository.upenn.edu/ese_papers

Part of the Engineering Commons, and the Physics Commons

\section{Recommended Citation}

Francisco J. Rodríguez-Fortuño and Nader Engheta, "Avoiding Metallic Walls: Use of Modal Superposition in Plasmonic Waveguides to Reduce Propagation Loss", . March 2012.

Rodríguez-Fortuño, F. J. and Engheta, N. (2012). Avoiding metallic walls: Use of modal superposition in plasmonic waveguides to reduce propagation loss. Physical Review B, 85(11), 115421. doi: 10.1103/PhysRevB.85.115421 @2012 American Physical Society

This paper is posted at ScholarlyCommons. https://repository.upenn.edu/ese_papers/605

For more information, please contact repository@pobox.upenn.edu. 


\title{
Avoiding Metallic Walls: Use of Modal Superposition in Plasmonic Waveguides to Reduce Propagation Loss
}

\author{
Abstract \\ We theoretically explore the possibility of reducing propagation loss in a metal-insulator-metal (MIM) \\ waveguide, using mode combinations to achieve wall-avoiding field distributions along a certain \\ propagation length. We present analytical results for several waveguides showing notable loss reduction, \\ and we discuss the trade-offs between low loss and high confinement present in this technique. \\ Disciplines \\ Engineering | Physics \\ Comments \\ Rodríguez-Fortuño, F. J. and Engheta, N. (2012). Avoiding metallic walls: Use of modal superposition in \\ plasmonic waveguides to reduce propagation loss. Physical Review B, 85(11), 115421. doi: 10.1103/ \\ PhysRevB.85.115421 \\ (C2012 American Physical Society
}




\title{
Avoiding metallic walls: Use of modal superposition in plasmonic waveguides to reduce propagation loss
}

\author{
Francisco J. Rodríguez-Fortuño ${ }^{1,2}$ and Nader Engheta ${ }^{1, *}$ \\ ${ }^{1}$ Department of Electrical and Systems Engineering, University of Pennsylvania, Philadelphia, Pennsylvania 19104, USA \\ ${ }^{2}$ Nanophotonics Technology Center, Universidad Politécnica de Valencia, 46022 Valencia, Spain \\ (Received 13 September 2011; revised manuscript received 1 February 2012; published 16 March 2012)
}

\begin{abstract}
We theoretically explore the possibility of reducing propagation loss in a metal-insulator-metal (MIM) waveguide, using mode combinations to achieve wall-avoiding field distributions along a certain propagation length. We present analytical results for several waveguides showing notable loss reduction, and we discuss the trade-offs between low loss and high confinement present in this technique.
\end{abstract}

DOI: 10.1103/PhysRevB.85.115421

PACS number(s): 41.20.Jb, 42.25.Bs, 42.25.Hz, 73.20.Mf

\section{INTRODUCTION}

The use of plasmonic waveguides to guide electromagnetic energy enables high field confinement; ${ }^{1-6}$ however, this comes at the expense of high dissipation due to ohmic losses in the metal. $^{7-9}$ The reduction of material and propagation losses is a topic of great interest in the plasmonics and metamaterials communities. ${ }^{10-17}$ In this article, we explore a method for such loss reduction based on multimode interference, which is a known technique ${ }^{18}$ in the field of nanophotonics. In particular, we were inspired by the approach of Popovic et al. for reducing losses in arrays of silicon waveguide crossings. ${ }^{19,20}$ In their work, they use a low-loss Bloch wave combination that is judiciously designed to avoid the walls of the silicon waveguide periodically at the crossings. The technique is useful for single crossings as well. ${ }^{21-23}$ In this paper, we propose an analogous concept, but for metal-insulator-metal (MIM) plasmonic waveguides. Multimode behavior in plasmonic waveguides has been observed ${ }^{24}$ and studied for useful devices, ${ }^{25,26}$ but not for the purpose we explore here. In this paper, we propose the use of a linear combination of modes in a plasmonic waveguide to achieve cancellation or reduction of the fields within the metallic walls and thus reduce power dissipation due to material losses in the transmission of energy from point $A$ to point $B$ along such a plasmonic waveguide. The losses of the combination of modes are not simply the sum of the losses of each individual mode because ohmic dissipation depends on the square of the currents in the metal and thus does not behave linearly. We aim to find the proper conditions under which we can minimize such power dissipation for sending electromagnetic power from point A to point B along this waveguide.

To illustrate the concept intuitively, we first start by considering a simple, finite-conductivity, parallel-plate waveguide with simple analytical expressions, and then we consider a realistic MIM waveguide at optical frequencies. Figure 1 shows the geometry of interest.

\section{FINITE-CONDUCTIVITY PARALLEL-PLATE WAVEGUIDE}

To tackle our simplified waveguide problem, we use a perturbation method: First we calculate the fields inside the parallel-plate waveguide assuming lossless perfect electric conductor walls; this gives us a set of well-known orthogonal modes (TEM, $T M_{i}$, and $T E_{i}$, where the integer $i$ refers to the number of half-wavelengths along the width of the waveguide) that we can combine linearly. Only then, using the perturbation method, do we estimate the losses by calculating the currents produced by the total fields in the metallic walls. The electric current in the metallic walls is given by $\mathbf{J}_{s}=\hat{\mathbf{n}} \times \mathbf{H}$, where $\hat{\mathbf{n}}$ is the unit outward vector normal to the walls To achieve the cancellation of $\mathbf{H}$, we must combine modes whose magnetic fields are not orthogonal to each other (i.e., we do not combine a $T M_{i}$ with a $T E_{i}$ mode). Restricting ourselves, for example, to the simple combination of only two modes-TEM and a $T M_{i}$-such that $\mathbf{H}=H_{y} \hat{\mathbf{y}}$, if one adds the field expressions for both modes and then computes the power loss at the conducting walls as $P_{L C}(z)=\left(R_{s} / 2\right)\left(\left|\mathbf{J}_{\mathbf{s}}\right|_{x=-a / 2}^{2}+\left|\mathbf{J}_{\mathbf{s}}\right|_{x=a / 2}^{2}\right)$, where $R_{s}=\left(\omega \mu(2 \sigma)^{-1}\right)^{1 / 2}$ is the surface resistance of the conductor with conductivity $\sigma, \omega$ is the angular frequency, and $\left|\mathbf{J}_{\mathbf{s}}\right|_{x=x_{0}}^{2}=\left|H_{y}\right|_{x=x_{0}}^{2}$, one arrives at the following expression for the losses in the conductor $P_{L C}\left(W \times m^{-2}\right)$ when $i$ is an even integer (so that both modes TEM and $T M_{i}$ have the same $H_{y}$ parity):

$$
\begin{aligned}
P_{L C}(z)= & P_{L C T E M}+P_{L C T M i} \\
& +2 \sqrt{P_{L C T E M} \times P_{L C T M i}} \cos (\Delta k z) .
\end{aligned}
$$

$P_{L C T E M}=\left(2 R_{S} / a Z_{T E M}\right) P_{T E M}^{+}$and $\quad P_{L C T M i}=\left(4 R_{S} /\right.$ $\left.a Z_{T M i}\right) P_{T M i}^{+}$are the losses for the TEM and $T M_{i}$ modes individually, $\quad Z_{T E M}=(\mu / \varepsilon)^{1 / 2}$ and $Z_{T M i}=\left(k_{z i} / \omega \varepsilon\right)^{1 / 2}$ are the impedances of these modes, $P_{T E M}^{+}\left(W \cdot m^{-1}\right)$ and $P_{T M i}^{+}\left(W \cdot m^{-1}\right)$ are the powers carried by the TEM and $T M_{i}$ modes, respectively, $P_{T O T}^{+}=P_{T E M}^{+}+P_{T M i}^{+}$, and $\Delta k=$ $k_{z T E M}-k_{z T M i}=k_{0}\left(1-\left(1-\left(f_{c T M i} / f\right)^{2}\right)^{1 / 2}\right)$. We note that the average power loss of this mode combination is equal to the sum of the power loss of the two modes propagating individually, but locally, the power loss varies with $z$. Because we can make $2 \sqrt{P_{L C T E M} \times P_{L C T M i}}$ equal to (but never greater than) $P_{L C T E M}+P_{L C T M i}$, the value of power dissipation can become identically zero at a certain point $z_{0}$ along the waveguide if we fulfill the condition $P_{\text {LCTMi }}=P_{\text {LCTEM }}$, which can also be written as

$$
P_{T M i}^{+} / P_{T E M}^{+}=\frac{1}{2} \frac{Z_{T M i}}{Z_{T E M}}=\frac{1}{2} \sqrt{1-\left(f_{c T M i} / f\right)^{2}} .
$$




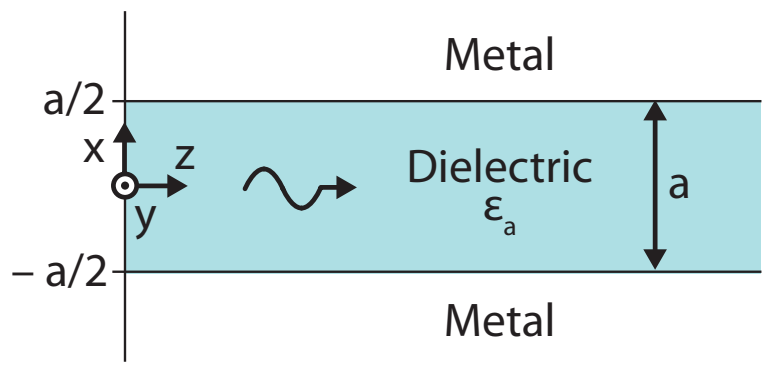

FIG. 1. (Color online) Geometry of the metal-insulator-metal (MIM) waveguide.

This condition achieves something interesting: Exactly at $z_{0}$, the free electrons in the metallic walls are not experiencing any forces at all from the electromagnetic fields that are being guided between the two walls; thus, locally, this guiding is not generating any ohmic losses. Figure 2 shows the field distribution and power flow of such a combination of modes $T E M$ and $T M_{2}$ under Condition (2), together with the power loss at the conducting walls, $P_{L C}$. The power flow varies from being confined at the center of the waveguide at $z_{0}$ to flowing near the walls at $z_{0} \pm L_{2 \pi} / 2$. The spatial period of this periodic behavior is given by $L_{2 \pi}=2 \pi /\left(k_{z T E M}-k_{z T M 2}\right)$.

Equation (1) can be proved to be valid for any other combination of two $T M_{i}$ modes given that they have the same $H_{y}$ parity. On the other hand, if the combined modes have opposite $H_{y}$ parity, then we simply obtain $P_{L C}(z)=$ $P_{L C T M i}+P_{L C T M j}$. In that situation, the power dissipation "bounces" from one wall to another, but the sum for both walls is constant at any point (e.g., when combining TEM and $\left.T M_{1}\right)$.

Now imagine that we want to transfer energy through this waveguide. We can make use of the drastically reduced power dissipation region around $z_{0}$ to transfer energy from $\mathrm{A}\left(z_{0}-\right.$ $L / 2)$ to $\mathrm{B}\left(z_{0}+L / 2\right)$ over a length $L$ that must be small compared with $L_{2 \pi}$ (the beat length of the two modes). To do this, we should excite point A with the mode profile that corresponds to the appropriate mode combination at that point. The effective dissipated power by the $T E M+T M_{i}$ (even $i$ ) mode combination under Condition (2), for a length $L$ around

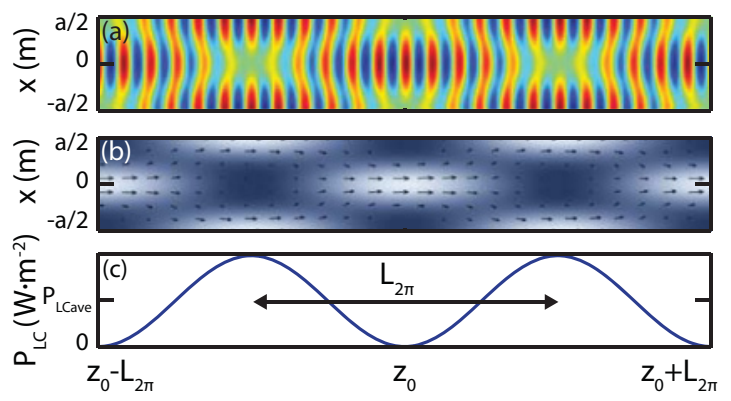

FIG. 2. (Color online) (a) $H_{y}$ field of the linear combination of $T E M$ and $T M_{2}$ modes under Condition (2) assuming perfect electric conductor walls. (b) Poynting vector. (c) Dissipated power if the metal walls have finite conductivity. $z_{0}$ can be defined and calculated as

$$
\begin{aligned}
P_{L C \text { eff }}(L) & \equiv \frac{1}{L} \int_{z_{0}-L / 2}^{z_{0}+L / 2} P_{L C}(z) d z \\
& =\left(P_{L C T E M}+P_{L C T M}\right)\left[1-\operatorname{sinc}_{\pi}\left(\frac{\Delta k L}{2 \pi}\right)\right],
\end{aligned}
$$

where $\operatorname{sinc}_{\pi}(x)=\sin (\pi x) / \pi x$ and we can rewrite $\Delta k L / 2 \pi=L / L_{2 \pi}=(L / \lambda)\left[1-\sqrt{1-\left(f_{c T M i} / f\right)^{2}}\right]$, where $\lambda=\left(c_{0} / f\right) \varepsilon_{r a}^{-1 / 2}$. The first term is the sum of the losses of the two modes individually, but clearly the second term can be very small if $L$ is sufficiently small compared with $L_{2 \pi}$, so that reduced losses are achievable. To obtain a fair insight into the improvement offered by this technique, we should compare the dissipated power $P_{L C \text { eff }}$ under Condition (2) with the smallest achievable dissipated power at the conductor using a single mode in the parallel-plate waveguide, which is by using the TEM mode to carry all the power $P_{T O T}^{+}$ by itself. Such comparison yields the following loss ratio $\left(L R=P_{L C \text { eff }}^{\text {Condition(2) }} / P_{L C T E M}^{P_{T E M}^{+}=P_{T O T}^{+}}\right)$:

$$
\begin{aligned}
L R= & {\left[\frac{2}{1+(1 / 2) \sqrt{1-\left(f_{c T M i} / f\right)^{2}}}\right] } \\
& \times\left\{1-\operatorname{sinc}_{\pi}\left[\frac{L}{\lambda}\left(1-\sqrt{1-\left(f_{c T M i} / f\right)^{2}}\right)\right]\right\} .
\end{aligned}
$$

Using this simple expression, we can plot normalized graphs showing the loss ratio (LR) as a function of propagation length $L$ and width of the waveguide $a$ both normalized to $\lambda$, as seen in Fig. 3. Notice that this graph is independent of the frequency and of the metal conductivity, so it can be regarded as a general result.

We see that significant loss reduction over a propagation distance of many wavelengths can be achieved if we work at high values of $\left(f / f_{c T M i}\right)$. For example, by combining TEM and $T M_{2}$ in a waveguide with $a=10 \lambda$, we can achieve a loss ratio of less than 0.1 (i.e., more than $90 \%$ reduced loss) from point $\mathrm{A}$ to B separated by a length $L=40 \lambda$. These results can readily be applied at microwave or terahertz frequencies. As we note,

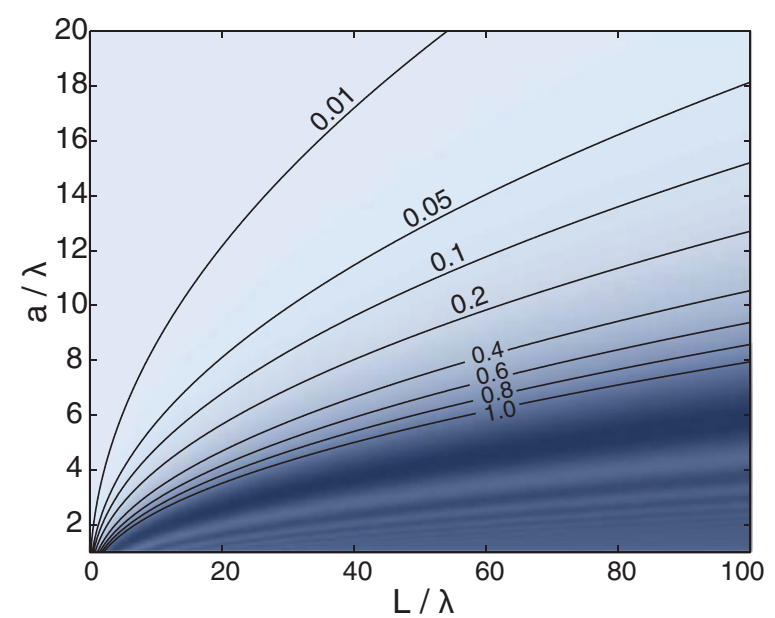

FIG. 3. (Color online) Loss ratio (LR) as a function of propagation length $L / \lambda$ and waveguide width $a / \lambda$ for a combination of TEM and $T M_{2}$ modes. In general, for a $T M_{i}$ mode, the vertical axis can be changed to $f / f_{c T M i}=(i / 2)(a / \lambda)$. 
it is desirable for $f / f_{c T M i}$ to be high (which translates into an electrically wide waveguide) because this means that the $T M_{i}$ mode is well above its cutoff frequency and therefore has a very similar propagation constant to that of the TEM mode, achieving a long beat length $L_{2 \pi}$ and therefore a long region of small losses around $z_{0}$. We will see that this trade-off between small waveguide width and low losses is present throughout all our results.

\section{MIM PLASMONIC WAVEGUIDE}

We now extend this study to the case of the MIM plasmonic waveguide with the aim of working at optical (infrared and visible) frequencies. In this case, the electromagnetic fields penetrate into the metal, and the currents in the metal are given by $\mathbf{J}=\sigma_{m} \mathbf{E}$, where $\sigma_{m}$ is the conductivity, so our aim is to achieve cancellation of the $\mathbf{E}$ field inside the metal using a linear combination of modes. The same symmetry considerations still apply. We will consider TM electromagnetic modes in a MIM plasmonic waveguide, whose field distributions and dispersion relations are known. ${ }^{8}$ We use the following nomenclature: Each mode $i$ will have a given propagation constant $k_{z i}$ at a given frequency $\omega$. The variation of fields along the transverse $x$ direction will be of the type $\exp \left( \pm \alpha_{m i} x\right)$ and $\exp \left( \pm \alpha_{a i} x\right)$ in the metal or in the dielectric region, respectively, where $\alpha_{m i, a i}^{2}=k_{z i}^{2}-\omega^{2} \mu \varepsilon_{m, d}$. The mode amplitude will be proportional to a scaling factor $M_{i}=H_{y}\left(x=a / 2, z=z_{0}\right)$. For the MIM geometry, only two modes exist below the dielectric light cone corresponding to the even and odd plasmonic modes (real $\alpha_{a i}$ ), whereas an infinite number of even and odd modes exist above the dielectric light cone, which shows trigonometric variation in the $x$ direction (imaginary $\alpha_{a i}$ ), which we refer to as dielectric slab modes. To cancel out or reduce the fields in the metal, we can choose to combine any two modes, as long as they have the same parity. Again, we use a perturbation method: We first calculate the two modes at a given frequency assuming lossless plasmonic metal (considering only a real part in the permittivity of metal $\varepsilon_{m}^{\prime}$ ). Then, we add the two mode's fields and estimate the losses of the total fields by taking into account the imaginary part of the metal permittivity $\varepsilon^{\prime \prime}{ }_{m}$. This method is valid only as long as the imaginary part of the permittivity does not substantially alter the mode's field profiles (i.e., that the perturbed lossy modal shapes are sufficiently similar to those of the lossless modes). To check this, we calculated that, in all the single modes used as examples in this paper, the estimated attenuation coefficient using the perturbation method described was within a $0.1 \%$ error of the exact attenuation coefficient obtained from an exact solution of the modes with a complex permittivity metal. For all our calculations, we take the values from Johnson and Christy $\varepsilon_{m}=\varepsilon_{m}^{\prime}+i \varepsilon_{m}^{\prime \prime}$ for silver. ${ }^{27}$ According to the perturbation method, the losses in the metal are evaluated using

$$
P_{L M}=\frac{\omega \varepsilon_{m}^{\prime \prime}}{2}\left(\int_{a / 2}^{\infty}|\mathbf{E}|^{2} d x+\int_{-a / 2}^{-\infty}|\mathbf{E}|^{2} d x\right)
$$

which for a single mode $i$ (with field amplitude $M_{i}$ and propagation constant $k_{z i}$ ) can be calculated by substituting the fields of a single mode into Eq. (5), resulting in

$$
P_{L M i}=\frac{\omega \varepsilon_{m}^{\prime \prime}}{2 \alpha_{m i}}\left(\frac{\left|M_{i}\right|}{\omega \varepsilon_{m}^{\prime}}\right)^{2}\left(k_{z i}^{2}+\alpha_{m i}^{2}\right) .
$$

When linearly combining two modes $i=1$ and 2, we first add the fields for the modes and then we apply Eq. (5), so after some mathematical manipulation, we arrive at the following expression for the power dissipation of the mode combination, which is the main result of our work:

$$
\begin{aligned}
P_{L M}(z)= & P_{L M 1}+P_{L M 2}+2 \sqrt{P_{L M 1} P_{L M 2}} \eta_{\alpha} \eta_{x z} \\
& \times \cos (\Delta k z+\Delta \phi) .
\end{aligned}
$$

where $P_{L M 1}$ and $P_{L M 2}$ are given by Eq. (6), $\eta_{\alpha}=$ $2\left(\alpha_{m 1} \alpha_{m 2}\right)^{1 / 2}\left(\alpha_{m 1}+\alpha_{m 2}\right)^{-1} \in[0,1], \quad \eta_{x z}=\left(\alpha_{m 1} \alpha_{m 2}+k_{z 1} k_{z 2}\right)$ $\left(\alpha_{m 1}^{2} \alpha_{m 2}^{2}+k_{z 1}^{2} k_{z 2}^{2}+\alpha_{m 1}^{2} k_{z 2}^{2}+\alpha_{m 2}^{2} k_{z 1}^{2}\right)^{-1 / 2} \in[0,1], \Delta k=k_{z 2}-$ $k_{z 1}$, and $\Delta \phi=\angle M_{2}-\angle M_{1}$. Equation (7) can be shown to be valid for any combination of two $T M$ modes in this geometry with the same parity. It is similar to the near perfect conductor case of Eq. (1), but now two terms, $\eta_{x z}$ and $\eta_{\alpha}$, prevent $P_{L M}\left(z_{0}\right)$ from becoming identically zero. The coefficient $\eta_{x z}$ arises because there are two components of the currents in the metal, $x$ and $z$, whose ratio is different for the two different modes, so having only one free parameter to tune (the relative power between the modes), we cannot exactly cancel both components simultaneously. The coefficient $\eta_{\alpha}$ takes into account that the rate of evanescent decay of fields into the metal is slightly different for both modes so the field cancellation cannot be identically zero for all regions $|x|>a / 2$. In practice, however, both coefficients are very close to 1 . Looking at Eq. (7), we can deduce that optimization of losses at $z_{0}$ takes place when $P_{L M 1}=P_{L M 2}$, which, in accordance with Eq. (6), happens when $\left|M_{2}\right| /\left|M_{1}\right|=\sqrt{\alpha_{m 2} \alpha_{m 1}^{-1}\left(k_{z 1}^{2}+\alpha_{m 1}^{2}\right)\left(k_{z 2}^{2}+\alpha_{m 2}^{2}\right)^{-1}}$. This simple and analytically exact condition guarantees minimum

TABLE I. Individual mode and mode combination parameters for the two examples, A and B.

\begin{tabular}{lcc}
\hline \hline & A (narrow waveguide) & B (wide waveguide) \\
\hline$a(\mu \mathrm{m})$ & 0.8 & 6.0 \\
$\lambda_{0}(\mu \mathrm{m})$ & 0.6 & 0.6 \\
\hline Mode 1 & Even & First even \\
& plasmonic mode & dielectric slab mode \\
$k_{z 1}\left(\operatorname{rad} \mu \mathrm{m}^{-1}\right)$ & 16.3 & 15.18 \\
$\alpha_{z 1}\left(\mathrm{~dB} \mu \mathrm{m}^{-1}\right)$ & 0.275 & $0.159 \cdot 10^{-3}$ \\
\hline Mode 2 & First even & Second even \\
& 14.0 & dielectric slab mode \\
$k_{z 2}\left(\operatorname{rad} \mu \mathrm{m}^{-1}\right)$ & 0.0867 & 15.10 \\
$\alpha_{z 2}\left(\mathrm{~dB} \mu \mathrm{m}^{-1}\right)$ & & $1.33 \cdot 10^{-3}$ \\
\hline$M_{\text {Mode } \operatorname{combination}}$ & 3.175 & 0.119 \\
$P_{\bmod e 2}^{+} / P_{\bmod e 1}^{+}$ & 0.9991 & 1.0000 \\
$\eta_{x z}$ & 1.0000 & 1.0000 \\
$\eta_{\alpha}\left(\operatorname{rad} \mu \mathrm{m}^{-1}\right)$ & 3.3 & 0.08 \\
$\Delta k_{z}\left(\mathrm{~dB} \mu \mathrm{m}^{-1}\right)$ & 0.132 & $0.284 \times 10^{-3}$ \\
$\alpha_{\text {ave }}(\mu \mathrm{m})$ & 1.34 & 77.4 \\
$L_{2 \pi}\left(\mu \mathrm{m}^{2}\right.$ & 0.65 & 50.0 \\
$L_{\max }$ & & \\
\hline \hline
\end{tabular}


power dissipation at $z_{0}$. We can force this condition by appropriately tuning the relative power carried by each mode. The experimental excitation of the two modes with the appropriate amplitudes and phases might prove challenging. However, the mode profile that has to be excited at point $\mathrm{A}$ is concentrated near the center of the waveguide, so exciting the MIM waveguide with a Gaussian beam or a single-mode dielectric waveguide, similar to what is done in Refs. 3-6, could be possible ways to access the $z_{0}$ region experimentally.

We will consider two examples demonstrating the features of the proposed technique, both of which work in the visible spectrum $f=500 \mathrm{THz}\left(\lambda_{0} \approx 0.6 \mu \mathrm{m}\right)$, where $\varepsilon_{m}(500 \mathrm{THz})=-16.04+0.4327 i$ and both use silica as the dielectric $\left(\varepsilon_{a}=1.45^{2}\right)$. To gain some insight and allow easy comparisons, we first calculate a local attenuation coefficient $\alpha_{z}(z)=P_{L M}(z) / 2 P_{T O T}^{+}$, which for individual modes is a constant value and represents the power attenuation $P^{+}(z) \propto$ $\exp \left(-\alpha_{z} z\right)$, but which for the combination of the two modes has a sinusoidal variation in $z$ with period $L_{2 \pi}$, reaching a minimum of $\alpha_{\text {ave }}\left(1-\eta_{x z} \eta_{\alpha}\right) \approx 0$ at $z_{0}$ and a maximum of $\alpha_{\text {ave }}\left(1+\eta_{x z} \eta_{\alpha}\right) \approx 2 \alpha_{\text {ave }}$ at $z_{0} \pm L_{2 \pi} / 2$, where $\alpha_{\text {ave }}$ is the average value of the attenuation coefficient of the two modes, weighed by their relative power. Then, for a given mode
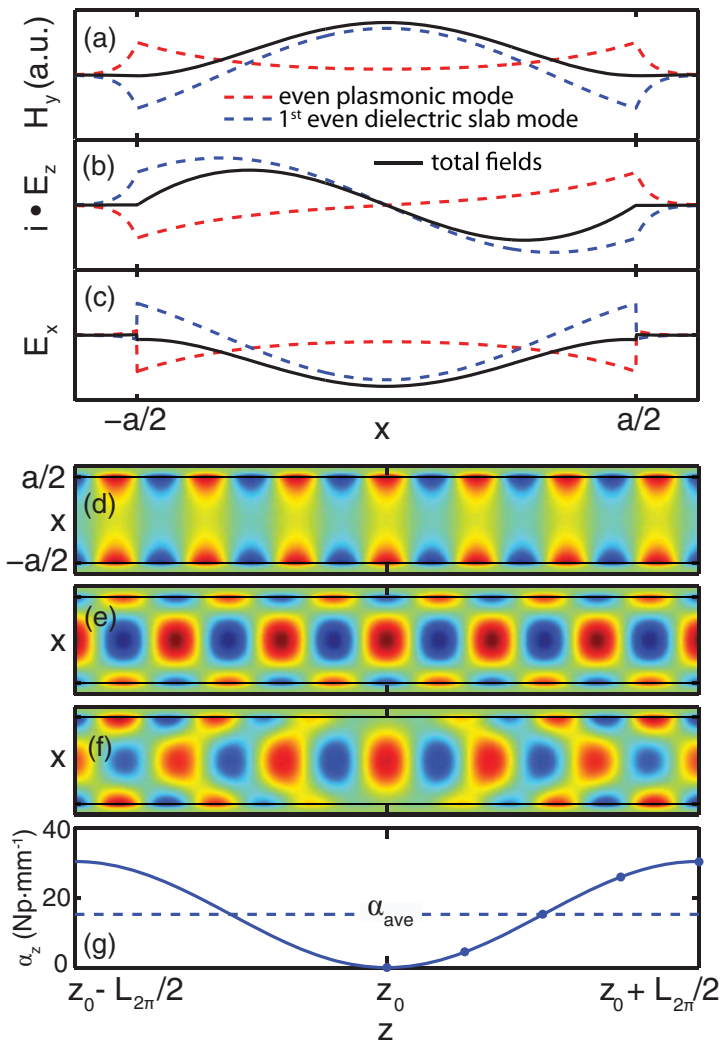

FIG. 4. (Color online) Electromagnetic field components (a) $H_{y}(x)$, (b) $i E_{z}(x)$, and (c) $E_{x}(x)$ at $z=z_{0}$ of both individual modes and the linear combination of both under the condition for minimized losses at $z=z_{0} . H_{y}(x, z)$ field distribution for (d) the even plasmonic mode, (e) the first even dielectric slab mode, and (f) the linear combination of both. (g) Local attenuation coefficient in the metal for the linear combination of modes. The continuous line represents the analytical calculation of Eq. (7), whereas the dots represent the loss numerically calculated from the field distribution. combination and a given propagation length $L$, we define an effective attenuation coefficient $\alpha_{\text {eff }}(L)=\frac{1}{L} \int_{z_{0}-L / 2}^{z_{0}+L / 2} \alpha_{z}(z) d z$, which if $\eta_{x z}$ and $\eta_{\alpha}$ are close to 1 , can be approximated by $\alpha_{\text {eff }}(L) \approx \alpha_{\text {ave }}\left[1-\operatorname{sinc}_{\pi}\left(L / L_{2 \pi}\right)\right]$. Additionally, it is interesting to compute the maximum propagation length $L_{\max }$ for which the use of the mode combination technique shows an improvement in terms of the total loss over any of the two constituent modes individually, that is, $L \leqslant L_{\max } \Rightarrow \alpha_{\text {eff }}(L) \leqslant$ $\min \left(\alpha_{z 1}, \alpha_{z 2}\right)$. All the relevant parameters for the two examples are summarized in Table I.

The first example, whose fields are shown in Fig. 4, is a narrow MIM waveguide where we adequately combine the even plasmonic mode with the first even dielectric slab mode to achieve reduction of the fields in the metal around $z_{0}$. The cancellation of fields inside the metal at $z_{0}$ is almost perfect. Unfortunately, in this case, $L_{\max }$ is not a very long distance relative to the wavelength because of the high $\Delta k_{z}$. Notice that we could use mode engineering with multilayered dielectrics between the two metals to further reduce $\Delta k_{z}$ and therefore increase $L_{\max }$.

Alternatively we can reduce $\Delta k_{z}$ by using more closely related modes. This is what we do in the second example, where we combine two dielectric slab modes on a wide waveguide so that their propagation constants are close to each other and to that of the dielectric, at the expense of lowering the confinement. This achieves a relatively large $L_{\max }=50 \mu \mathrm{m}$. So if we want to transfer energy on such a MIM waveguide for a distance $|L|<50 \mu \mathrm{m}$, the use of the appropriate mode combination as shown will reduce total loss with respect to using a single mode. In this case, the improvement can be very noticeable for smaller distances $L$; for example, if $L=10 \mu \mathrm{m}$, then the effective attenuation constant is as low as $\alpha_{\text {eff }}(10 \mu \mathrm{m})=7.73 d B \mathrm{~m}^{-1}$, considerably smaller than the $159 \mathrm{~dB} \mathrm{~m} \mathrm{~m}^{-1}$ of the lowest loss dielectric slab mode.

\section{CONCLUSIONS}

Using the linear combination of two modes in a MIM waveguide, we have found the condition under which the losses of the mode combination are minimized to almost zero at a given point in the waveguide. Transmission of energy for a length $L$ in the neighborhood of that point shows very low total power dissipation. Our study was performed both for good conductors, valid at microwave and terahertz frequencies, and for Drude-type metals at optical frequencies, yielding very similar conclusions. The reduction of losses comes however at the cost of reducing the high confinement typical of plasmonic modes. Because we require modes with geometrical variation inside the dielectric, the width of the waveguide must be comparable to the wavelength. Under such condition, one may conceivably use a dielectric waveguide altogether showing zero metal dissipation; however, the presence of metal can be useful for other purposes, such as electrodes in an electrooptical modulator. Therefore, our present work is useful for devices in which the metals are unavoidable or desirable.

\section{ACKNOWLEDGMENTS}

F. J. Rodríguez-Fortuño acknowledges financial support from grant FPI of $\mathrm{GV}$ and the Spanish MICINN under 
contracts CONSOLIDER EMET CSD2008-00066 and TEC2011-28664-C02-02. This work is supported in part by the
Office of Naval Research (ONR) Multidisciplinary University Research Initiative (MURI) grant No. N00014-10-1-0942. *engheta@ee.upenn.edu

${ }^{1}$ W. L. Barnes, A. Dereux, and T. W. Ebbesen, Nature 424, 824 (2003).

${ }^{2}$ J. A. Schuller, E. S. Barnard, W. Cai, Y. C. Jun, J. S. White, and M. L. Brongersma, Nat. Mater. 9, 193 (2010).

${ }^{3}$ E. Economou, Phys. Rev. 182, 539 (1969).

${ }^{4}$ C. Pfeiffer, E. Economou, and K. Ngai, Phys. Rev. B 10, 3038 (1974).

${ }^{5}$ J. Takahara, S. Yamagishi, H. Taki, A. Morimoto, and T. Kobayashi, Opt. Lett. 22, 475 (1997).

${ }^{6}$ S. A. Maier and H. A. Atwater, J. Appl. Phys. 98, 011101 (2005).

${ }^{7}$ P. Berini, Opt. Express 14, 13030 (2006).

${ }^{8}$ S. A. Maier, Plasmonics: Fundamentals and Applications (Springer, New York, 2010).

${ }^{9}$ E. Ozbay, Science (New York, NY) 311, 189 (2006).

${ }^{10}$ A. Boltasseva and H. A. Atwater, Science (New York, NY) 331, 290 (2011).

${ }^{11}$ D. A. Bobb, G. Zhu, M. Mayy, A. V. Gavrilenko, P. Mead, V. I. Gavrilenko, and M. A. Noginov, Appl. Phys. Lett. 95, 151102 (2009).

${ }^{12}$ R. F. Oulton, V. J. Sorger, D. A. Genov, D. F. P. Pile, and X. Zhang, Nature Photonics 2, 496 (2008).

${ }^{13}$ J. T. Kim, J. J. Ju, S. Park, M.-su Kim, S. K. Park, and S.-Y. Shin, Opt. Express 18, 2808 (2010).
${ }^{14}$ L. Chen, J. Shakya, and M. Lipson, Opt. Lett. 31, 2133 (2006).

${ }^{15}$ S. A. Maier, P. E. Barclay, T. J. Johnson, M. D. Friedman, and O. Painter, Appl. Phys. Lett. 84, 3990 (2004).

${ }^{16}$ D. Dai and S. He, Opt. Express 18, 17958 (2010).

${ }^{17}$ M. Wu, Z. Han, and V. Van, Opt. Express 18, 11728 (2010).

${ }^{18}$ L. B. Soldano and E. C. M. Pennings, J. Lightwave Technol. 13, 615 (1995).

${ }^{19}$ M. A. Popovic, University of Colorado Boulder (personal communication).

${ }^{20}$ M. A. Popovic, E. P. Ippen, and F. X. Kartner, LEOS 2007-IEEE Lasers and Electro-Optics Society Annual Meeting Conference Proceedings 56 (2007).

${ }^{21}$ H. R. Stuart, Opt. Lett. 28, 2141 (2003).

${ }^{22}$ H. Liu, H. Tam, P. Wai, and E. Pun, Opt. Commun. 241, 99 (2004).

${ }^{23}$ H. Chen and A. W. Poon, IEEE Photonics Technol. Lett. 18, 2260 (2006).

${ }^{24}$ B. Steinberger, A. Hohenau, H. Ditlbacher, A. L. Stepanov, A. Drezet, F. R. Aussenegg, A. Leitner, and J. R. Krenn, Appl. Phys. Lett. 88, 094104 (2006).

${ }^{25}$ Y.-J. Tsai, A. Degiron, N. M. Jokerst, and D. R. Smith, Opt. Express 17, 17471 (2009).

${ }^{26}$ Y. Kou and X. Chen, Opt. Express 19, 6042 (2011).

${ }^{27}$ P. B. Johnson and R. W. Christy, Phys. Rev. B 6, 4370 (1972). 